\title{
THYMOMA: A RARE CAUSE OF MEDIASTINAL TUMOR IN CHILDREN
}

\author{
Andreea Durlan', Cristina Oana Marginean ${ }^{2}$, Maria Despina Baghiu', \\ Alina Grama ${ }^{2}$, Emoke Horvath ${ }^{3}$, Mihaela-Ioana Chincesan ${ }^{2}$ \\ ${ }^{1}$ Pediatrics Clinic I, Emergency County Hospital, Targu Mures \\ ${ }^{2}$ Pediatrics Discipline, University of Medicine and Pharmacy, Targu Mures \\ ${ }^{3}$ Pathology Discipline, University of Medicine and Pharmacy, Targu Mures
}

\begin{abstract}
Thymoma is a thymic neoplasm composed of epithelial cells, rarely seen in children. Approximately two-thirds of mediastinal tumors in children are asymptomatic. The diagnosis is suspected following the compression of the tumor mass on the airways. We present the case of a male patient, age 7 years and 8 months, who presented at the emergency room accusing fatigue at rest or minimum effort, dry cough, intense dyspnea, orthopnea, tachypnea, chest pain, being transferred to the Pediatric Cardiology Clinic in Tirgu Mures with the following diagnoses: fluid pericarditis, right pleural effusion and cardiac tamponade. Chest computer tomography examination is carried out, describing a solid mass of 100/89/111 mm in the anterior mediastinum, partially incorporating the thymus and the vascular structures of the middle mediastinum. Pericardectomy is practiced with partial removal of the tumor and extensive tumor cytoreduction and the histopathologic examination is B3 Thymoma. Specific treatment is initiated according to the Tymoma VENUTA protocol, followed by adjuvant radiotherapy, with clinical and radiological remission. The particularity of the case is the fact that thymoma is a rare tumor on the patient's age, creating diagnostic difficulties, has no complications during treatment, with favorable evolution, with clinical and radiological remission at 1.9 years after.
\end{abstract}

Keywords: child, thymoma, mediastinal tumor

\section{INTRODUCTION}

The thymus is a gland shaped like the letter $\mathrm{H}$, occupying the anterior mediastinum. It covers the pericardium and the large vessels at the base of the heart (1). Thymic tumors are the most frequently resected tumors from the mediastinum, but they are rarely in children less than 16 years old (2). They are frequently (95\%) located in the anterior compartment of the mediastinum, but can be found at the neck, lung or left pulmonary hilum $(1,3)$.

Epithelial and stromal tissues of the thymus are influencing the selection and maturation of $\mathrm{T}$ lymphocytes and impaired lymphocyte selection process leads to abnormal cell proliferation. Thymoma is usually an encapsulated tumor (benign non-invasive thymomas), $70 \%$ is associated with paraneoplastic syndromes such as myasthenia gravis, aplastic anemia, collagen diseases, pemphigus and immunodeficiency $(3,4)$. Benign thymoma can be- come highly vascularized with bleeding or necrotic tissues, but can be large and asymptomatic for long periods. Malignant thymomas are rarely invading (metastasizing) the surrounding tissues - such as lungs, pericardium or large blood vessels, but with very serious consequences. The compression syndromes involve the bronchi, lungs or superior vena cava syndrome (SVCS). It may occur at the local spread of benign thymoma, thymic cysts or thymic carcinoma (3). Symptoms may include chest pain, superior vena cava syndrome, dyspnea, dysphagia and cough (4). Peripheral blood lymphocyte immunophenotyping shows an absent or very low number of B cells and decreased T lymphocytes CD4 + (2). Treatment consists of surgical resection, radiotherapy and postoperative chemotherapy for invasive thymoma, and it is reported to have a favorable response rate of $70-80 \%(3,4)$. Given that thymoma is usually well encapsulated, thymectomy can be curative in early stages.

Corresponding author:

Cristina Oana Marginean, University of Medicine and Pharmacy, 38 Gh. Marinescu Street, Targu Mures, Romania

E-mail: marginean.oana@gmail.com 
The prognosis after surgical resection varies depending on the histology, regional and distant invasion of the tumor. The survival rate associated with encapsulated thymomas is $97 \%$ at 5 years (7).

\section{CASE PRESENTATION}

We present a male patient, aged 7 years and 8 months, whose personal pathological history mention a rare dry cough in the last 2 months. He is presenting one day prior hospitalization dry cough, fatigue at rest or minimum effort, intense dyspnea, orthopnea, tachypnea, chest pain. He presents at the territorial emergency room, where after chest X-ray and cardiac ultrasound, he is urgently transferred at the Pediatric Cardiology Clinic of Targu Mures with the diagnoses of fluid pericarditis, cardiac tamponade and right pleural effusion requiring surgical drainage. At the Pediatric Cardiology Clinic, the 24 hours evolution is unfavorable and he is transferred at the Cardiovascular Surgery Clinic. Chest CT scan with intravenous contrast describes a solid mass 100/89/111 mm size, with cystic areas, developed in the anterior mediastinum, incorporating the thymus and partially the vascular structures of the middle mediastinum. The described mass wraps the superior vena cava and is also coming into contact with the pericardium, extending up to the upper cranial part of the sternal manubrium and caudal at $1.5 \mathrm{~cm}$ from the diaphragm (Fig. 1. a, b, c, d). Pericardectomy is practiced with partial removal of the tumor, extensive tumor cytoreduction,
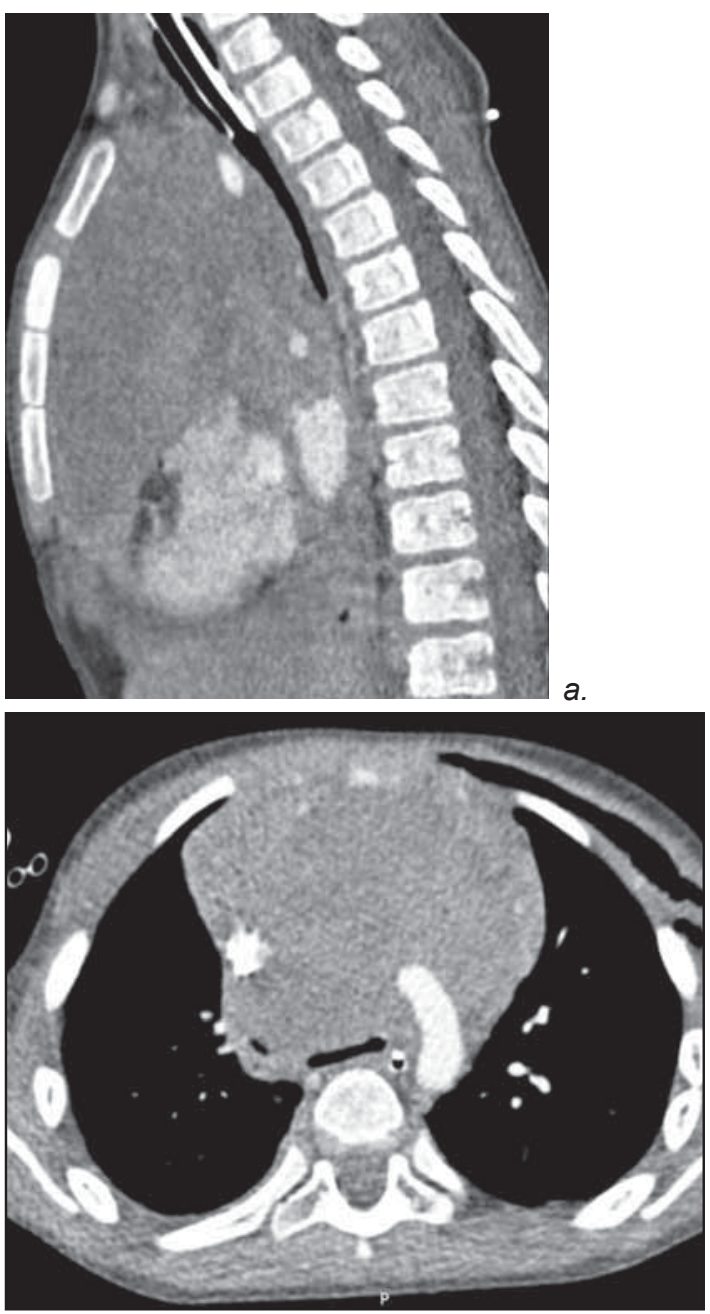

C.
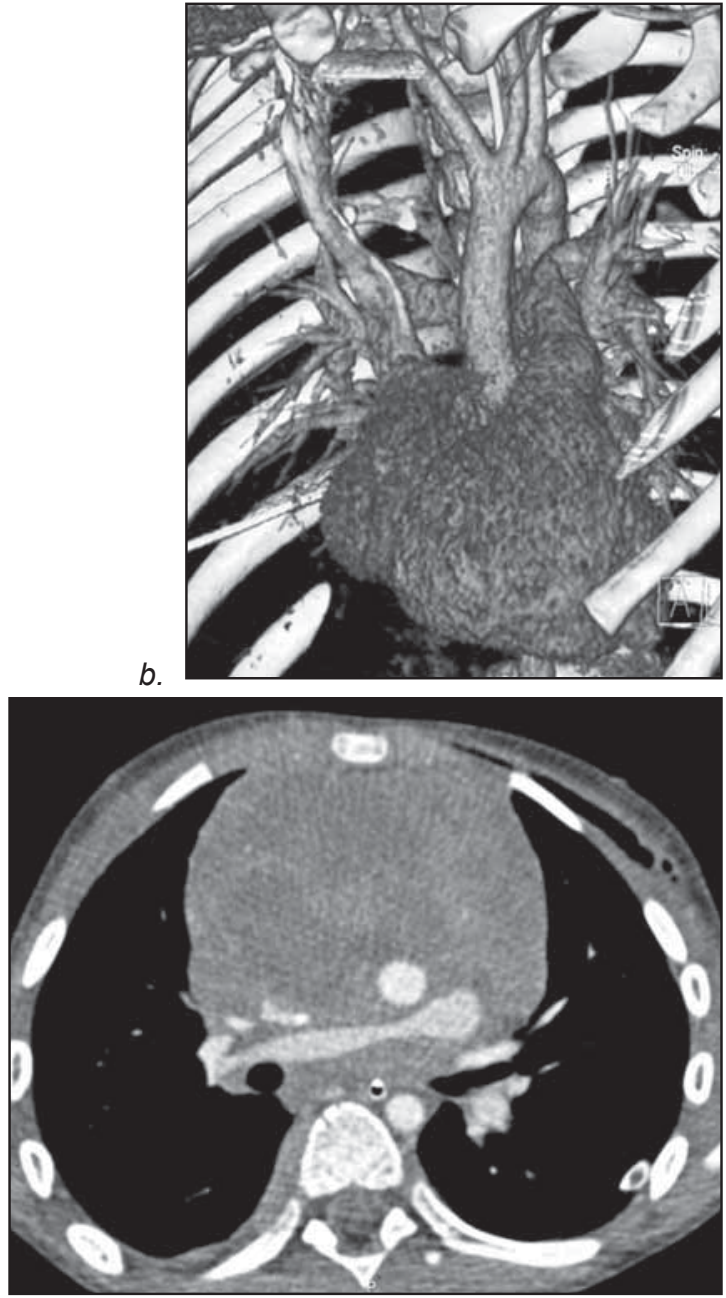

d.

\section{FIGURE 1.}

a. CT image reconstruction, sagital postcontrast iv - bulky mass of solid consistency, heterogeneous with moderate iodophily, occupying the anterior mediastinum, wraping the great vessels and pushing them posteriorly, marked compression of the trachea.

b. CT post contrast iv image (3D reconstruction) anterior and oblique view - Highlighting deviation and compression on the great vessels (aorta and SVC).

c. Iv post contrast CT axial section - infiltrative mass encompassing the large vessels pushing them posterior and tracheomalacia.

d. Infiltrative mass that causes compression on the vessels - SVC and right pulmonary artery. 
and decompression of the superior caval vein system and the respiratory tree segment. The fragments are sent for histopathological examination. On day 7 of the disease, he is transferred in the Hemato-Oncology Department of Pediatric Clinic I. Physical examination reveals malaise, pale skin, toracotomy scar above the chest, generalized lymphadenopathy. Laboratory tests on admission reveal anemia (Hemoglobin 9.5 g/l); LDH (lactic dehydrogenase): 1,468 U/1; Positive acute phase reactants; peripheral smear with $74 \%$ segmented neutrophils, hypersegmented polymorphonuclears; bone marrow with normal celularity; cerebrospinal fluid without modification; immunogram within normal limits; Neuron specific enolase elevated $(143,7 \mathrm{ng} / \mathrm{ml})$; Vanilmandelic acid/24 hour urine - normal, increased ferritin $(191 \mathrm{ng} / \mathrm{ml})$ and Copraemia within normal limits. Imaging tests were performed (CT thorax-abdomen-pelvis with iv. contrast), highlighting the remaining of a dense mass in the anterior mediastinum (probable thymic tissue), nodes located in the Baretty lodge of $23 \mathrm{~mm}$, no retroperitoneal lymph nodes detected, parenchymal organs homogeneous before and after contrast, moderate hepatosplenomegaly. Ultrasound revaluation reveals a minimum blade of fluid in the pericardium and blade of fluid in the right pleura of $1.3 \mathrm{~cm}$; biventricular impaired relaxation, diastolic dysfunction and normal biventricular systolic function. The provisory histopathological result was T-cell Lymphoblastic Lymphoma, but TDT enzyme (terminal deoxynucleotidil transferase) specific for thymus $\mathrm{T}$ lymphocytes raised the suspicion of other pathology. For this reason, to obtain the correct diagnosis, the paraffin block was sent to the Pathology Department of Budapest Institute of Oncology. Meanwhile oncologic treatment is initiated (ALL IC-BFM 2009), then the result of the histopathological examination is B3 Thymoma. Following the review of the patient status, chemotherapy is initiated according to the protocol VENUTA Tymom.

Two months later after the chemotherapy, he followed adjuvant radiotherapy (DT 40 gray/20 fr.). 4 months after completion of radiotherapy he was without pathological FDG (fludeoxyglucose) fixation on PET/CT. The review conducted after one year declares clinical and radiological remission, and the last PET/CT 1.9 years after the treatment completion is negative.

\section{DISCUTIONS}

Thymoma and thymic carcinomas are epithelial tumors of the thymus. In children, thymic tumors represent only $2-3 \%$ of all mediastinal tumors, thy- moma is very rare $(<1 \%)(5)$. A third of patients with thymoma are diagnosed as a result of an abnormality on a chest X-ray, such as widening of the mediastinum at the anteroposterior incidence or retrosternal clouding at the side incidence (3). In the presented case, chest X-ray was performed due to respiratory symptoms, suspecting a cardiac tamponade. But chest CT or MRI can reveal the morphology of the tumor mass, presence of cysts or necrosis. When performing the CT scan, the presented patient had a solid mass in the anterior mediastinum with cystic areas, incorporating the thymus and partially the vascular structures of the middle mediastinum, wrapping also the superior vena cava. Although less frequently, metastases occur in $30-40 \%$ of patients with advanced disease $(3,7)$. The literature concludes that approximately $70 \%$ of patients with thymoma have symptoms of other diseases, including myasthenia gravis $(50 \%)$, hypogammaglobulinemia (5\%), aplastic anemia $(5 \%)$, immune or endocrine disorders (10\%) (2). The patient presented above has not developed any of these pathologies, the only manifestation of the disease being given by local compression. Surgical resection is the main treatment for thymic epithelial lesions. Radiotherapy and chemotherapy are reserved for the treatment of invasive and malignant types (5).

A recent comparative study (153 patients, Werneck et al) found no statistical difference between patients where thymectomy was performed and the other group of patients who received conservative treatment (6). Having signs of cardiac tamponade and superior vena cava compression, our patient required emergency surgery for the decompression of superior caval vein system and respiratory segment. The prognosis of these patients depends on the presence or absence of metastases or tracheal compression, vascular complete excision, histologic type epithelial, lymphocyte type or mix, tumor size greater than $8 \mathrm{~cm} \mathrm{(1).}$

\section{CONCLUSIONS}

Although thymoma is a rare tumor, diagnosis must be considered in front of a chest radiograph with a widened mediastinum, associated with signs of superior vena cava compression and respiratory symptoms. Loco-regional tumor invasion, without distant metastases resulted in a favorable response to oncological therapy, followed by radiotherapy. The prognosis is favorable, considering the last clinical review, biological and imaging performed. 


\section{REFERENCES}

1. Plesa C. Surgical pathology of the thymus. In: Barza M., Crumpei F., Danila N., Filip V., Fotea V. General Surgery Vol II. Iasi: Sakura Publishing 2001:372-377

2. Xiayuan L., Mark AL., Kelley E.C. et al. Thymoma in Children: Report of 2 Cases and Review of the Literature. Pediatr Dev Pathol 2010; 13: 202 -208

3. Meena P., Vaishali A., Sujit J. Thymoma in a child - A case report. Indian J Med Res vol 2 2013; 7:721-725

4. Hamidah A., Poulsaeman V., Suria AA., et al. Thymoma a case report and review of the literature. Med and Health 2010; 5(1):49-54
5. Santana L., Givica A., Camacho C. Best cases from the AFIP: Thymoma. Armed For Inst Pathol 2002; 22:S95-102

6. Wernek L., Cunha F., Scola R. Myasthenia Gravis: Clinical evaluation of 153 patients. Acta Neural Scand 2000; 101:41-46.

7. Carretto E., Inserra A., Ferrari A. et al. Epithelial thymic tumors in paediatric age, a report from the TREP project. Orphanet $J$ Rare Dis $2011 ; 6: 28$ 